\title{
Numerical Analysis for the Progressive Failure of Binary-Medium Interface under Shearing
}

\author{
Rihong Cao, ${ }^{1,2}$ Wenyu Tang, ${ }^{1}$ Hang Lin $\left(D,{ }^{1}\right.$ and Xiang Fan ${ }^{3}$ \\ ${ }^{1}$ School of Resources and Safety Engineering, Central South University, Changsha, Hunan 410083, China \\ ${ }^{2}$ School of Civil, Environmental, and Mining Engineering, The University of Western Australia, Perth, WA 6009, Australia \\ ${ }^{3}$ School of Highway, Chang'an University, Xi'an 710064, China \\ Correspondence should be addressed to Hang Lin; linhangabc@126.com
}

Received 25 August 2017; Accepted 7 February 2018; Published 1 April 2018

Academic Editor: Robert Černý

Copyright (C) 2018 Rihong Cao et al. This is an open access article distributed under the Creative Commons Attribution License, which permits unrestricted use, distribution, and reproduction in any medium, provided the original work is properly cited.

Binary-medium specimens were fabricated using the particle flow code, and the shear strength, dilatancy, and failure behavior of the binary-medium specimens with different bond strength ratios $(0.25,0.5,0.75$, and 1.0$)$ under different normal stresses were studied. Numerical results show that the bond strength ratio and normal stresses considerably influence the shear strengths of binary-medium interface. Shear strength increases as the bond strength ratio and normal stress increase. The dilation of interfaces with high bond strength ratios is more evident than those of interfaces with lower bond strength ratios, and the curves for the high bond strength ratio exhibit remarkable fluctuations during the residual stage. At increased normal stress and bond strength ratio, the peak dilation angle shows decreasing and increasing trends successively. In this study, the specimens exhibited three kinds of failure modes. In mode II, the sawtooth experienced shear failure, but some tensile cracks appeared on the interface of the binarymedium. In mode III, no sawtooth was cut off, indicating tensile failure on the interface. At a low bond strength ratio, damage or failure is mostly concentrated in the upper part of the model. Failure parts gradually transfer to the lower part of the model when the bond strength ratio and normal stress increase. Furthermore, evident tensile cracks occur on the interface. When the bond strength ratio reaches 1.0, the failure mode of the specimen gradually transforms from sheared-off failure to chip-off failure. The number of microcracks in the specimens indicates that the lower the bond strength ratio, the more severe the damage on the specimens.

\section{Introduction}

Contact problem between different materials is often encountered in geotechnical engineering [1-4]. Such problem is mostly encountered between concrete dam and rock foundation, anchor mortar and rock surface, and concrete pile and rock or soil [5-7]. The contact interface between two different materials is usually called a binary-medium interface [8-10]. The mechanical properties of the interface of a binary-medium are complicated because of the different materials involved. Furthermore, the binary-medium interface is a weak surface that has a significant impact on the stability of rock mass under complex stress environments. For example, parts on both sides of the interface rock or concrete medium produce relative sliding in a compressshearing stress state and damage the continuity and integrity of the material, thereby causing engineering damage under a long-term load action. Many studies on the interface in rock mass engineering start on the discontinuous surface of rock mass and most of them are based on the direct shear test under constant normal stress. The results of the study showed that the mechanical properties of rock joints are affected by the normal stress, joint surface roughness, and inherent mechanical properties of rocks [11-14]. Several current models for the estimation of joint shear strength exist, including the Coulomb model, Patton model, Barton model, and Byerlee model. Using these models, many researchers have made some amendments to obtain better results [15-19]. Theoretical derivation and experimental research are the most common research methods of rock mechanics and engineering. In light of the continuous development and progress of computer technology in recent 


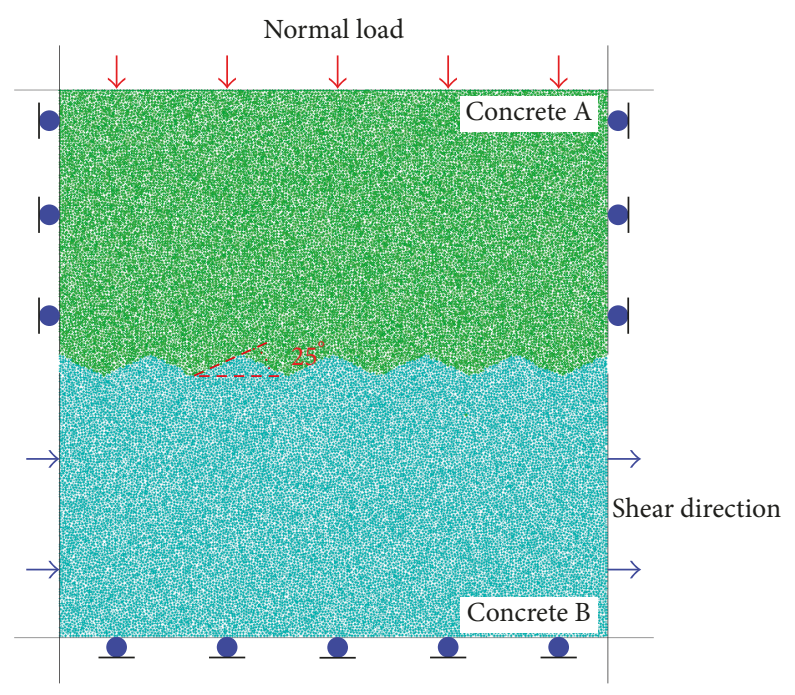

FIGURE 1: Binary-medium specimens.

TABLE 1: Microscopic parameters for rock mass.

\begin{tabular}{lcccccc}
\hline $\begin{array}{l}\text { Contact } \\
\text { modulus }(\mathrm{GPa})\end{array}$ & $\begin{array}{c}\text { Contact stiffness } \\
\text { ratio }\end{array}$ & $\begin{array}{c}\text { Bond radius } \\
\text { coefficient }\end{array}$ & $\begin{array}{c}\text { Bond modulus } \\
(\mathrm{GPa})\end{array}$ & $\begin{array}{c}\text { Bond stiffness } \\
\text { ratio }\end{array}$ & $\begin{array}{c}\text { Normal bond } \\
\text { strength }(\mathrm{MPa})\end{array}$ & $\begin{array}{c}\text { Shear bond } \\
\text { strength }(\mathrm{MPa})\end{array}$ \\
\hline 27 & 2.1 & 1.0 & 27 & 2.1 & 46 & 46 \\
\hline
\end{tabular}

years, researchers have developed a variety of numerical calculation methods on the basis of existing theoretical results and successfully used them to analyze the stability of rock mass engineering. Among these methods, the finite element, boundary element, and discrete element have been generally recognized. In the past decade, the discrete element method has been the most common numerical calculation method used to analyze rock failure and widely applied in research on cracking and failure in brittle rock-like materials. Furthermore, results of numerical simulation and laboratory and field experiments have shown good correlation [20-23]. In this paper, the numerical analysis was performed on direct shear tests, in which different bond strength ratios under different normal stress were used. In addition, shear strength parameters and interface failure process under different normal stresses were analyzed from the microscopic view through discrete element numerical analysis method PFC2D. The results can be used as reference for the prediction of failure face and strength of rock-like mass engineering.

\section{Numerical Model}

The binary-medium interface is a regular sawtooth, and the fluctuating angle is $25^{\circ}$. Figure 1 shows its shear numerical model, the lower part of which is high-strength concrete. The upper part represents low-strength concrete. The bond strength ratio $\left(R_{\mathrm{b}}\right)$ between the upper concrete (concrete $\mathrm{A}$ ) and lower concrete (concrete $\mathrm{B}$ ) is set to be $0.25,0.5,0.75$, and 1.0. When the ratio is 1 , the upper concrete and lower concrete have the same strength. In concrete with different proportions, the friction coefficient of the aggregate is basically consistent. In this paper, the particle flow code method was performed, and parallel-bond model was adopted. In this model, the particle is similar to fine sand, and the parallel bonds among particles are similar to cement. Thus, the particles are cemented into a solid mass. The direct shear test is used in the numerical simulations, and normal stress is applied to the upper part of the model at 1.0, 2.0, 3.0, and $4.0 \mathrm{MPa}$. The side wall of the upper part of the shear is supported by the rolling support boundary with the velocity of the wall, and the particles are maintained at $0.03 \mathrm{~mm} /\left(10^{6}\right.$ step. Meanwhile, the velocity from left to right is applied to the side wall of the lower part for transverse shear tests. In this study, the development of microcracks in the specimen was monitored by the fish program during the loading process.

In the lower part of the concrete (Table 1), the normal bond strength between the particles is consistent with the tangent bond strength. According to the microparameters of the lower part of the concrete (Table 1), normal bond strength is consistent with tangent bond strengths among the particles. The stress-strain curves and failure modes of the concrete specimens under uniaxial compression with different strength ratios are shown in Figure 2. The curve 4 shown in Figure 2(a) is a stress-strain curve with a bond strength ratio of 1 and peak strength of $60 \mathrm{MPa}$, which is also the strength of the lower concrete with high strength. The peak strength of the upper concrete (concrete A) varies with the change in the bond strength ratio. At increased bond strength ratios, the peak compressive strength of the sample increases, but the elastic modulus change is not evident at increased bond strength. In failure modes under different bond strengths, shear failure modes are observed in the specimens under different bond strengths, and obvious shear bands are detected in the specimens (Figure 2(b)). 


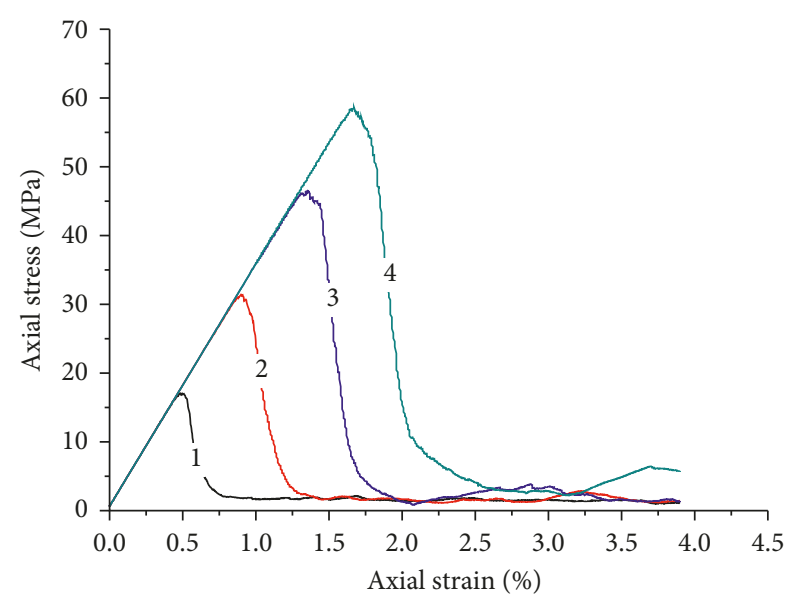

Bond strength ratio

$\begin{array}{ll}1-0.25 & 3-0.75 \\ 2-0.5 & 4-1.0\end{array}$

(a)

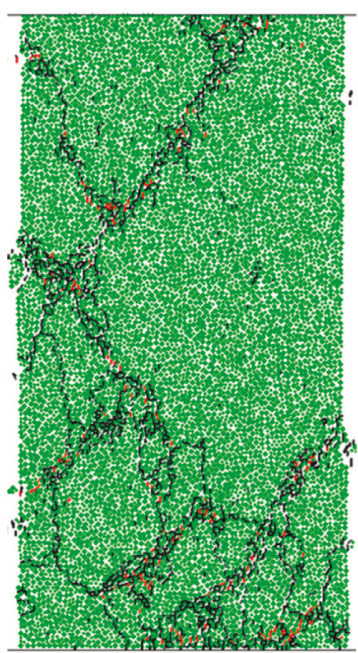

(b)

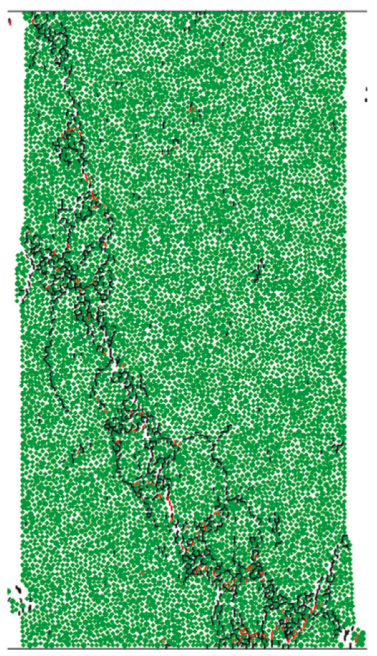

(d)

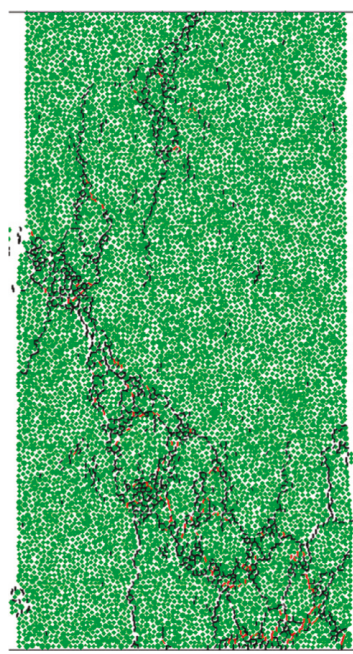

(c)

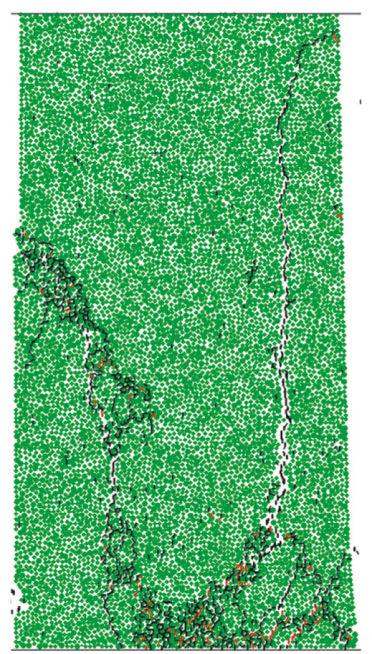

(e)
FIgURE 2: Strength and failure mode for specimen with different bond strength ratios: (a) the stress-strain curve. Failure mode for bond strength ratio is (b) 0.25 , (c) 0.50, (d) 0.75, and (e) 1.0 .

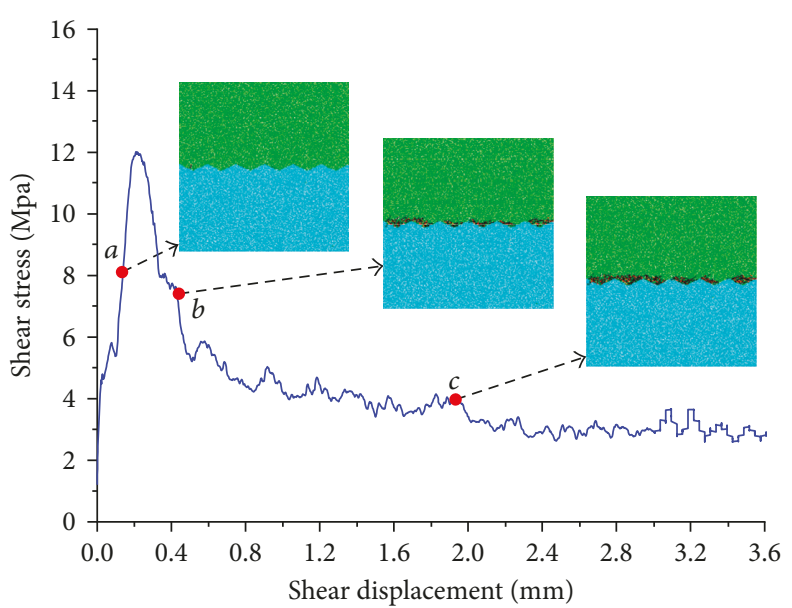

(a)

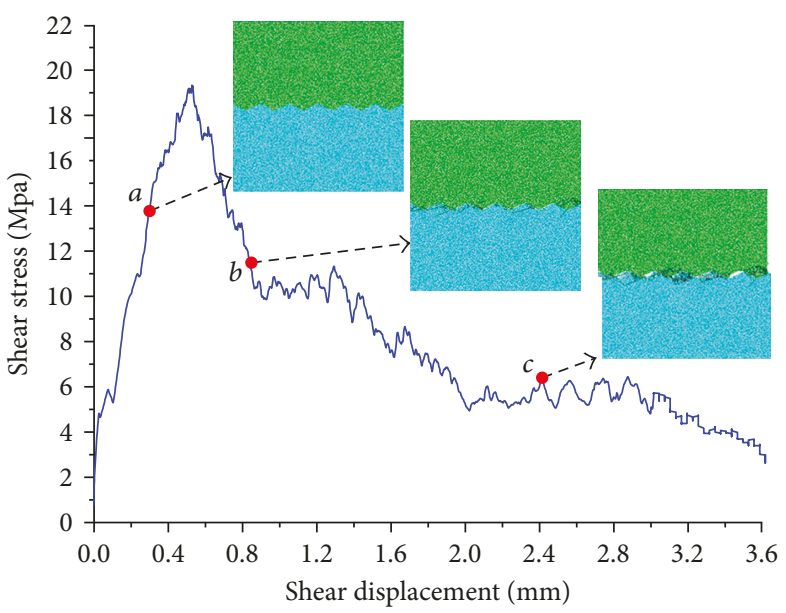

(b)

FIGURE 3: Shear stress and failure process for specimens with different bond strength ratios and normal stresses: (a) bond strength ratio of 0.25 and normal stress of $3.0 \mathrm{MPa}$; (b) bond strength ratio of $1.0 \mathrm{MPa}$ and normal stress of $3.0 \mathrm{MPa}$.

\section{Numerical Results}

3.1. Shear Failure Characteristics of the Binary-Medium Interface. Figure 3 shows the direct shear failures of binary-medium specimens with different bond strengths. The shear stress-displacement curve of the specimen with a bond strength ratio of 0.25 under a normal stress of 3.0 MPa and damages at different stages are shown in Figure 3(a). The shear curve is apparently flat after the peak value, indicating that the sample is destroyed gradually after the peak value and residual strength are reached rapidly. The point a on the shear curve represents the internal damage of the specimen prior to the peak value. A certain tensile failure around the binary-medium interface is present, but the specimen has no structural failure, and the asperities have not been cut off. When the shear curve reaches point $b$ during the declining stage, and the shear stress of the specimen decreases rapidly, the asperities at the upper part of the model have been cut off. The rapid decrease in shear stress indicates that the specimen has structural failure. The 

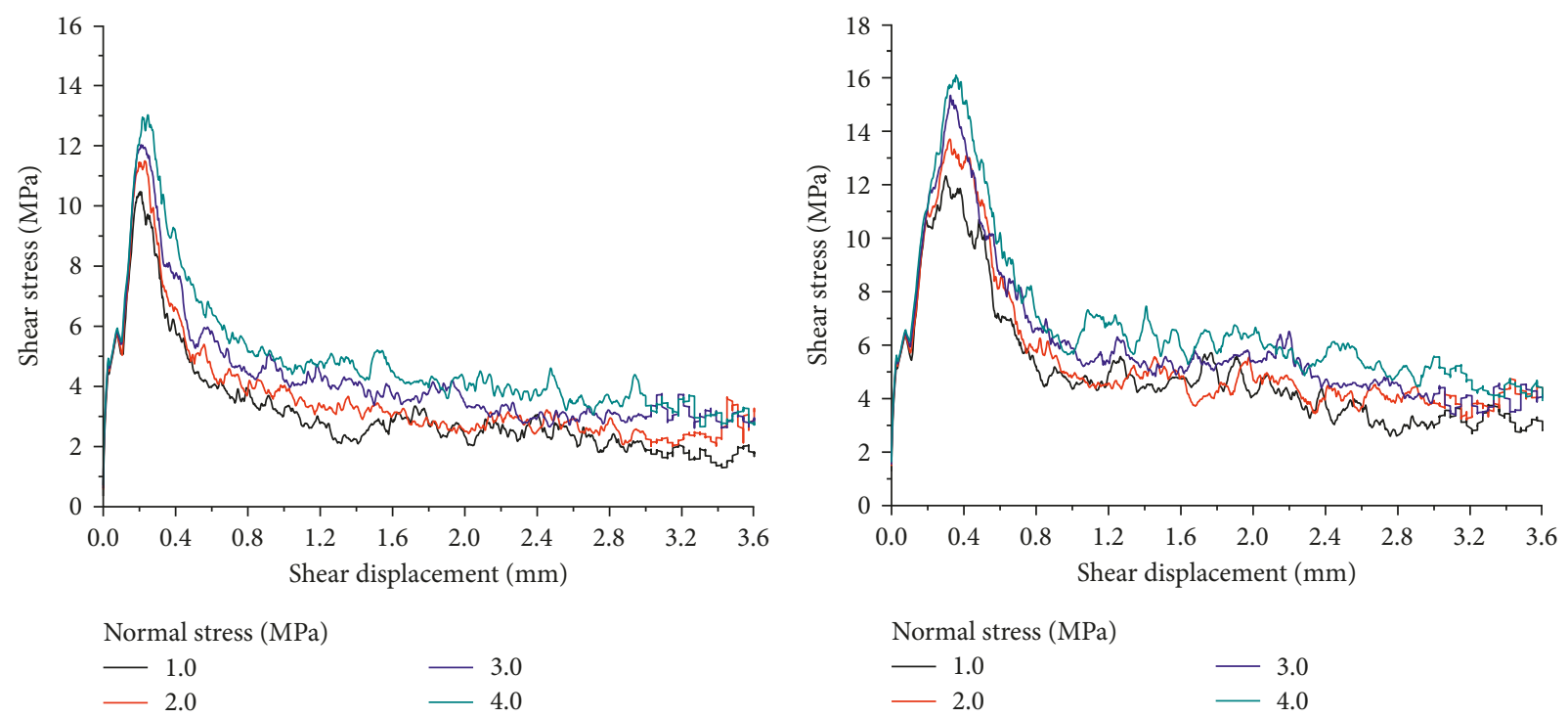

(a)

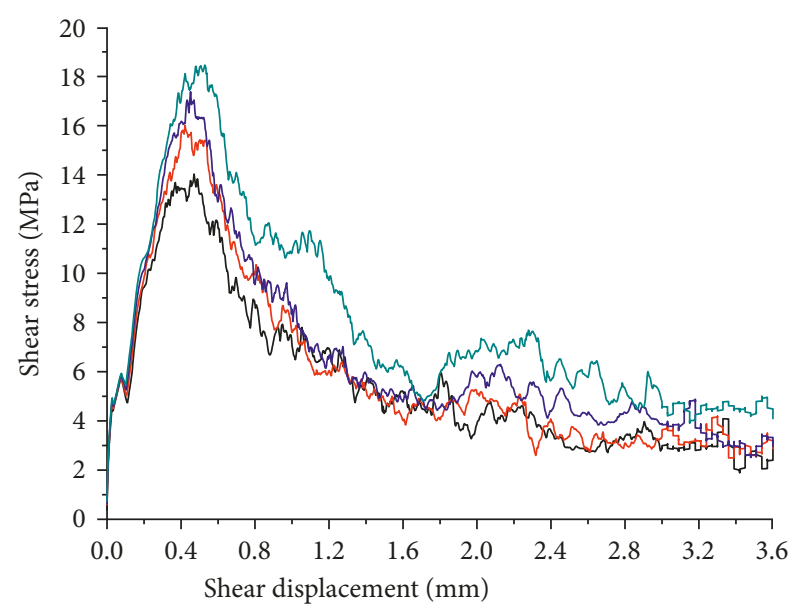

(b)

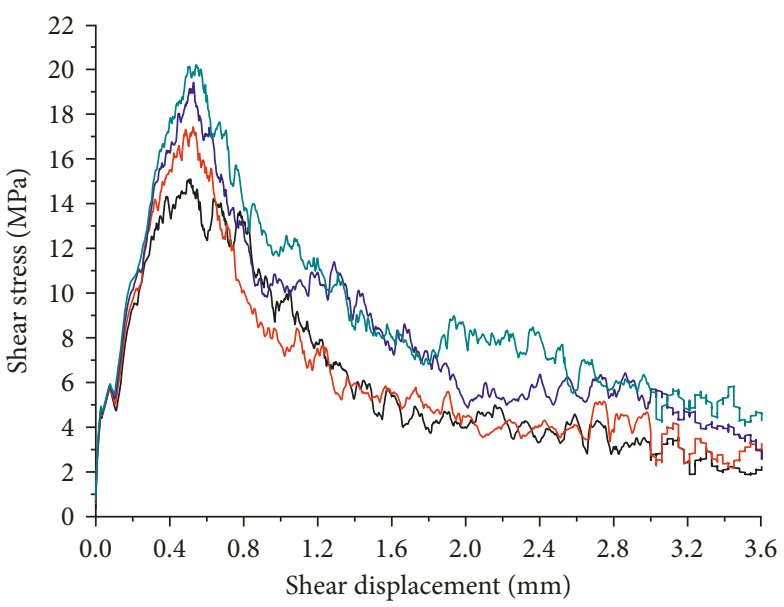

Normal stress (MPa)

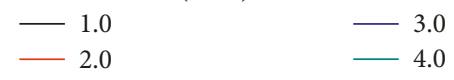

Normal stress (MPa)

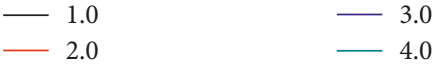

(d)

Figure 4: Shear curves for specimen with different bond strength ratios: bond strength ratios of (a) 0.25, (b) 0.5, (c) 0.75, and (d) 1.0.

shear stress-displacement curve has no apparent change in the residual stage as the shear simulation experiments are continued. Furthermore, the asperities on the upper part of the model are further sheared, and the straight shear surface is formed in the residual stage.

Figure 3(b) shows the shear stress-displacement curve of the specimen with a bond strength ratio of 1.0 under a normal stress of 3.0 MPa and internal damage. Shear stress declines sharply after its peak, similar to the shear stressdisplacement curve shown in Figure 3(a). However, unlike the shear stress that tends to flatten after decreasing (Figure $3(\mathrm{a})$ ), the shear stress shown in Figure $3(\mathrm{~b})$ is similar to a stepwise decline. In short, the fluctuation of the curve in Figure 3(a) is more evident than that in Figure 3(b), mainly because the asperities are sheared after the peak, while the initiation and propagation of the tensile crack in the residual stage leads to apparent fluctuations in shear stress. The higher the concrete strength, the more evident the fluctuation of the shear curve with invariant normal stress, and the different types of failure modes considerably affect strength parameters and shear curves.

\subsection{Shear Stress-Displacement Curves and Strength} Parameters. Figure 4 shows the shear stress-displacement curves with different bond strengths and normal stresses. Figures 4(a)-4(d) are the shear stress-displacement curves for the bond strength ratio of $0.25,0.5,0.75$, and 1.0 , respectively. The shear stress-displacement curves of the specimen nearly drop after they increase to the peak shear strength when shear displacement increases. The curves then flatten. Moreover, the peak shear stress of the specimen 
increases when the normal stress for the model with the same bond strength ratio increases, and the curves are consistent before the peak shear stress. At increased bond strength ratio, the ductility of the curve becomes more evident after the peak shear. Furthermore, neither the bond strength of concrete A nor the normal stress has a considerable influence on the residual stage of the curve. Peak shear displacement increases when the bond strength increases, while the increase in normal stress has no effect on peak shear displacement.

Figure 5 shows the relationship of the variations in peak shear strength and bond strength with bond strength and normal stress. As shown in Figure 5(a), the peak shear strength shows a significant linear increase at increased normal stress and bond strength. Four straight lines can be obtained by fitting the peak strength of the specimen with different bond strengths. Similar to rock mass, the shear strength of the binary-medium interface can also be expressed as follows:

$$
\tau=C+\sigma_{\mathrm{n}} \tan \varphi,
$$

where $\tau$ is the shear strength, $C$ is the cohesion of binarymedium interface, $\sigma_{\mathrm{n}}$ is the normal stress, and $\varphi$ is the friction angle of the interface.

Figure 5(b) shows the relationship between interface shear strength and bond strength ratio $\left(R_{\mathrm{b}}\right)$. Similar to the peak shear strength depicted in Figure 5(a), shear strength in Figure 5(b) has a linear growth at increased bond strength ratio $\left(R_{\mathrm{b}}\right)$. Figure $5(\mathrm{c})$ shows the variations in cohesion $C$ and slope $k=\tan \varphi$ with bond strength ratio. At increased concrete bond strength ratio $\left(R_{\mathrm{b}}\right)$, both parameters considerably increase. Furthermore, the antishear capacity of the binarymedium interface increases when the bond strength ratio increases. The relationship between the shear strength parameters and bond strength ratios of the binary-medium interface, shown by (2) and (3), respectively, is obtained by fitting the shear strength parameters:

$$
\begin{gathered}
C=a_{1}+b_{1} R_{\mathrm{b}}+b_{2} R_{\mathrm{b}}^{2}, \\
\phi=\arctan \left(a_{2}+b_{3} R_{\mathrm{b}}+b_{4} R_{\mathrm{b}}^{2}\right),
\end{gathered}
$$

where $C$ and $\varphi$ are the cohesion and friction angles of interface, respectively, and $R_{\mathrm{b}}$ is the bond strength ratio. The values of $a_{1}, a_{2}, b_{1}, b_{2}, b_{3}$, and $b_{4}$ are $7.417,0.29,10.17,-3.9$, 2.52 , and -1.112 , respectively, for this study.

3.3. Analysis of Dilatancy. When the strength of concrete A increases, the shear strengths and shear-displacement curves of the specimen show evident changes. During direct shear, concrete A has a shear dilation trend, and the growth and trend of shear dilation are affected by the strength of the material. For the analysis of the effect of the upper concrete bond strength on shear dilation, the normal displacement of the upper wall is monitored and calculated during the simulation, and the shear dilation displacement-shear displacement curve is shown in Figure 6. The four curves in

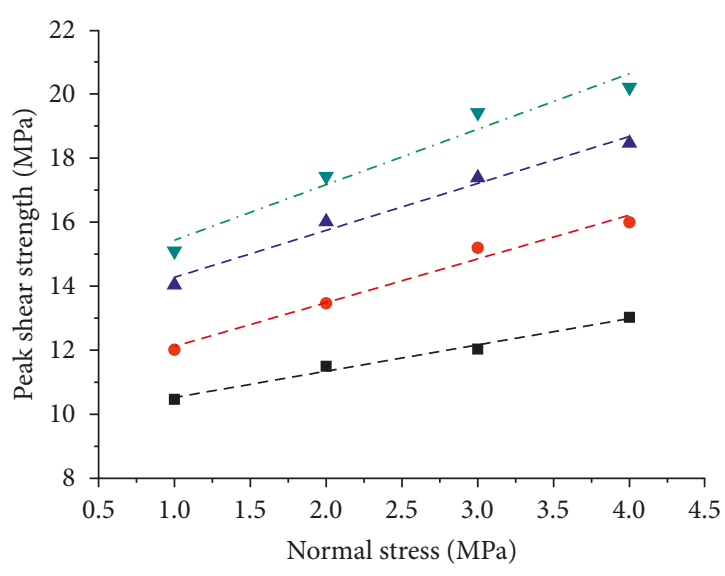

Bond strength ratio

- 0.25

- 0.5

A 0.75

$\checkmark 1.0$

(a)

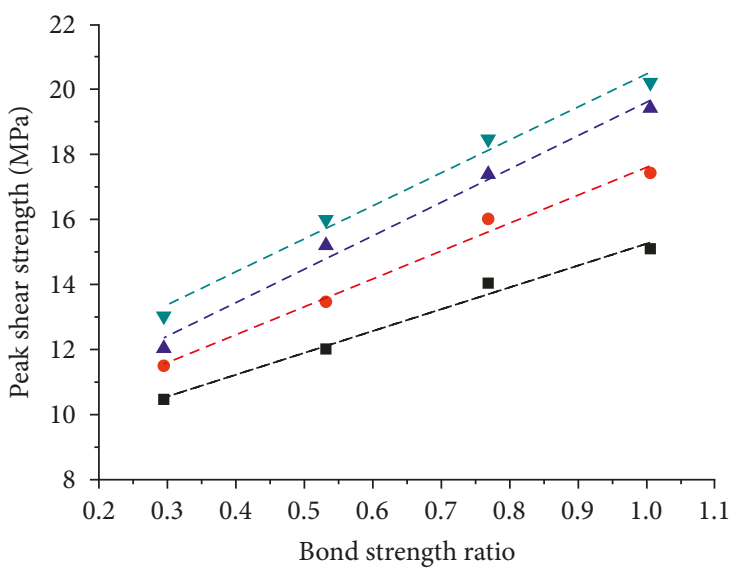
- 1.0
- 2.0
$\Delta 3.0$
$\checkmark 4.0$

(b)

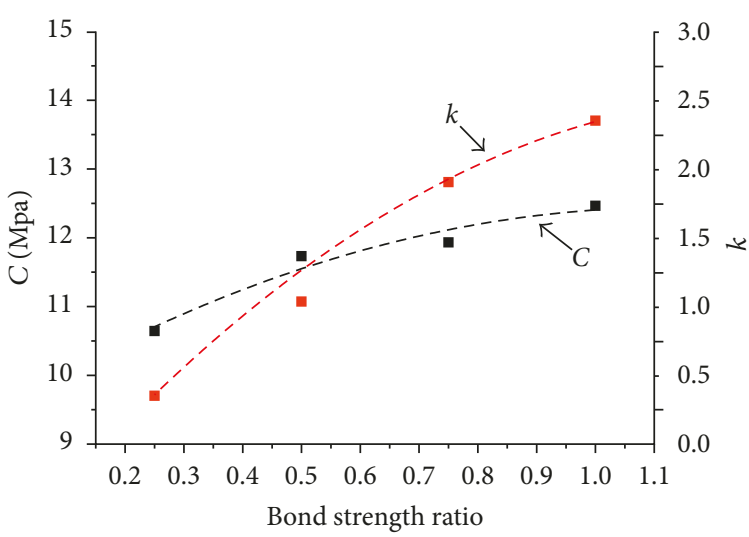

(c)

FIGURE 5: Relationship between the shear strength parameters and bond strength ratio: (a) relationship of peak shear strength and normal stress; (b) relationship of peak shear strength and bond strength ratio; (c) relationship of $C$ and $k$ with bond strength ratio. 

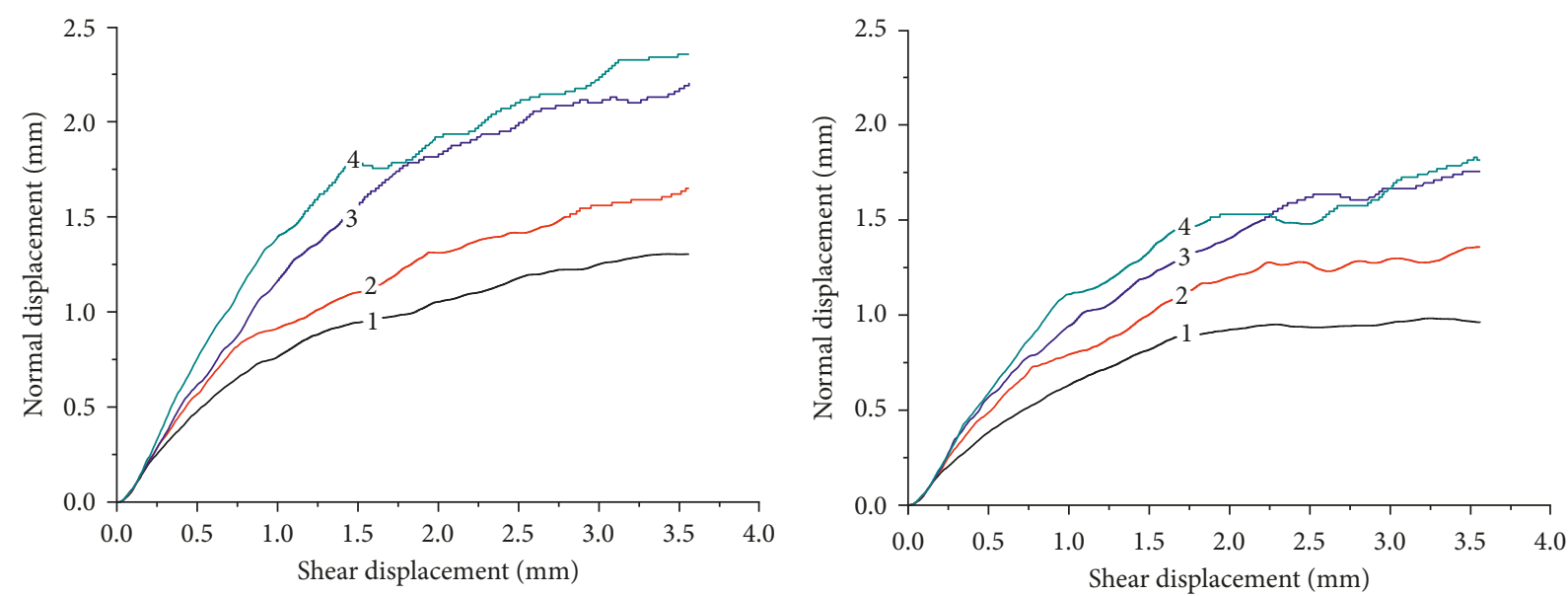

Bond strength ratio
$1-0.25$
$3-0.75$
$2-0.5$

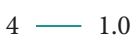

(a)

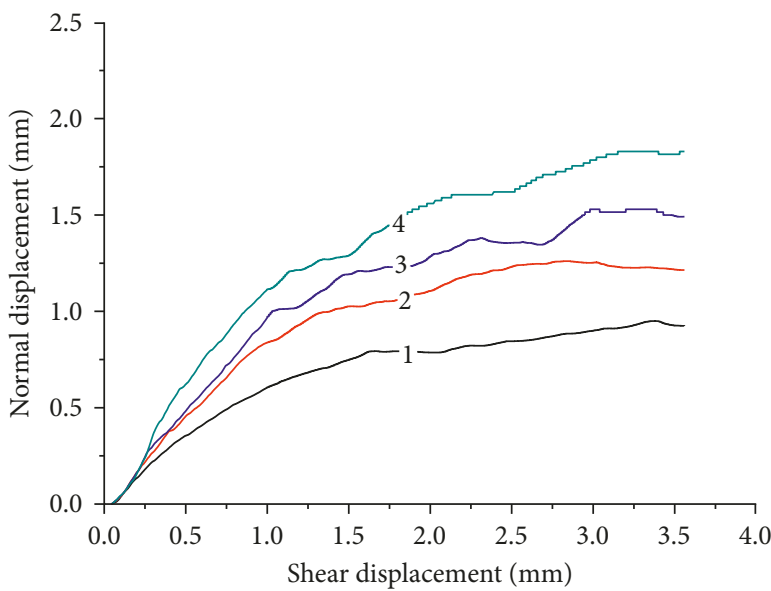

Bond strength ratio

$$
\begin{array}{ll}
1-0.25 & 3-0.75 \\
2-0.5 & 4-1.0
\end{array}
$$

(c)

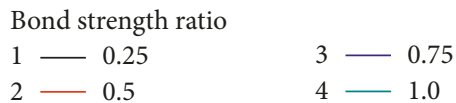

(b)

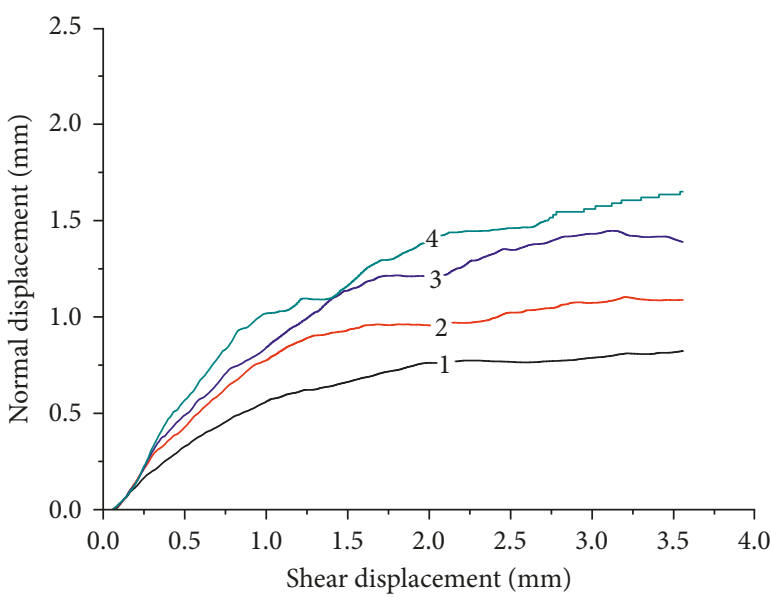

Bond strength ratio

$$
\begin{aligned}
& 1-0.25 \\
& 2-0.5
\end{aligned}
$$

(d)

FIGURE 6: Dilation curve for specimen with different bond strength ratios: normal stress of (a) $1.0 \mathrm{MPa}$, (b) 2.0 MPa, (c) $3.0 \mathrm{MPa}$, and (d) 4.0 MPa.

Figures 6(a) and 6(d) show a significant growth trend as the shear displacement increases. The curve 4 , representing the bond strength ratio of 1.0 , is located above the other curves, whereas the curve representing the bond strength ratio of 0.25 is at the bottom. This result indicates that the strength of concrete $\mathrm{A}$ is low, and the asperities on concrete A are easily destroyed by normal and shear loads. Thus, large degrees of sliding along the serrated surface cannot occur and form shear dilation after the asperities are partially cut. When the bond strength ratio is 0.25 , the shear expansion curves increase smoothly as shear displacement increases, which corresponds to the shear stress-displacement curve in Figure 4.

In the residual stress stage, shear stress tends to be gentle, indicating that shear dilation is caused by the broken pieces and resulting friction among them. These pieces are produced by the overall destruction of the specimen. As the bond strength ratio increases, the failure module of the binary-medium interface is not only the shear or friction failure in concrete A but also some friction failures and tensile crack propagations in concrete $\mathrm{B}$. Thus, the dilation curve fluctuates at increased shear displacement, which is not as smooth as the bond strength ratio of 0.25 . Notably, similar to the direct shear test, shear dilation decreases at increased normal stress and shear dilation reaches a minimum value at a normal stress of $4.0 \mathrm{MPa}$.

Similar to that of rock joint surface shear, different degrees of dilation similar to that of rock joint interface are observed during the shear test of binary-medium interface. The degrees of dilation and dilation angle vary with bond strength ratio. The dilation angle of joint surface is usually calculated by 


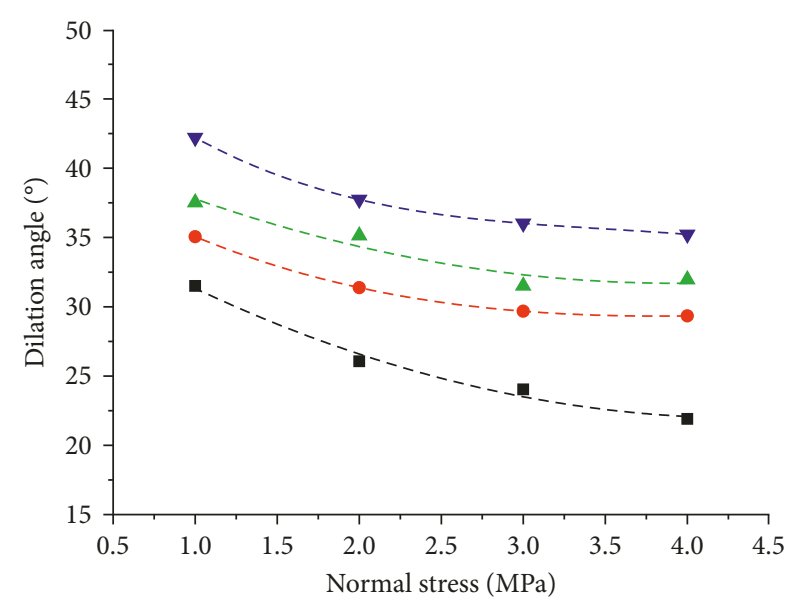

Bond strength ratio
- 0.25
^ 0.75
- 0.5
$\checkmark 1.0$

(a)

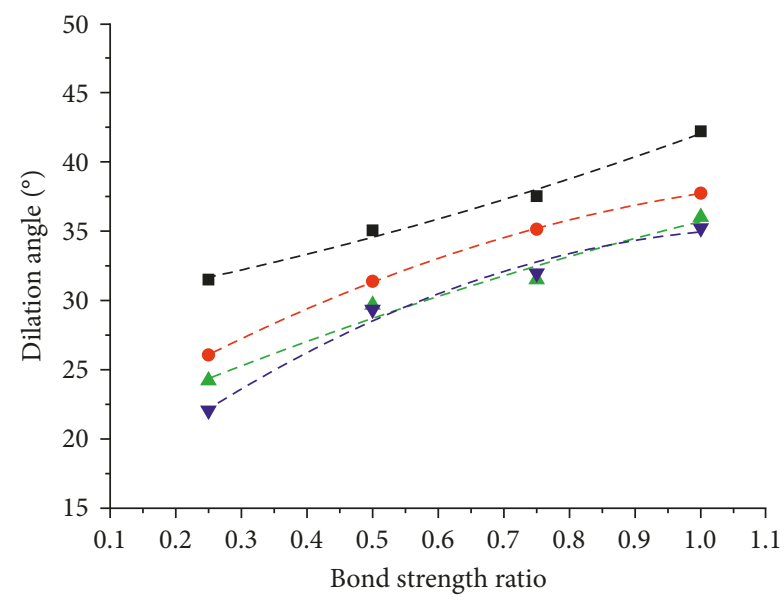

Normal stress (Mpa)

$\begin{array}{ll}\text { - } 1.0 & \boldsymbol{\Delta} 3.0 \\ -2.0 & \boldsymbol{\nabla} 4.0\end{array}$

(b)

FIgURE 7: Trends of the peak dilation angle: (a) relationship of dilation angle and normal stress; (b) relationship of dilation angle and bond strength ratio.

$$
\tan \psi=\frac{\Delta u_{\mathrm{v}}}{\Delta u_{\mathrm{h}}}
$$

where $\psi$ is the dilation angle and $\Delta u_{\mathrm{v}}$ and $\Delta u_{\mathrm{h}}$ are the normal and shear displacement increment, respectively.

The relationship between shear displacement and the tangent value of the dilation angle can be obtained after the normal displacement of each period is divided by shear displacement. Thus, we can extract the peak tangent value of the dilation angle of the different models with different normal stresses, and then we can deduce the change rule of the dilation angle. Figure 7 shows the change in the peak dilation angle of the binary-medium interface with the normal stress and the bond strength ratio. Figure 7 (a) shows
TABLE 2: Fitting parameters for peak dilation angle with different bond strength ratios.

\begin{tabular}{lcc}
\hline Bond strength ratio & $a$ & $b$ \\
\hline 0.25 & 31.434 & -0.257 \\
0.5 & 34.79 & -0.134 \\
0.75 & 37.65 & -0.13 \\
1.0 & 41.899 & -0.133 \\
\hline
\end{tabular}

TABLE 3: Fitting parameters for peak dilation angle with different normal stress.

\begin{tabular}{lccc}
\hline Normal stress $(\mathrm{MPa})$ & $a$ & $b_{1}$ & $b_{2}$ \\
\hline 1 & 29.336 & 8.1936 & 4.512 \\
2 & 19.474 & 29.136 & -10.892 \\
3 & 19.441 & 20.815 & -4.56 \\
4 & 13.738 & 37.909 & -16.7 \\
\hline
\end{tabular}

that the peak shear dilation angle of the binary-medium interface has a decreasing trend at increased normal stress as the strength of the normal constraints increases. Thus, the normal displacement is limited, and the peak shear dilation angle decreases gradually. The following formula is obtained by fitting the peak dilation curve:

$$
\psi=a \sigma_{\mathrm{n}}^{b}
$$

where $a$ and $b$ are the fitting coefficients and $\sigma_{\mathrm{n}}$ is the normal stress.

The fitting parameters for the peak dilation angle with different normal stress values are shown in Table 2. Figure 7(b) shows the relationships among the peak dilation angle, bond strength ratio, and fitting curve. Apparently, the peak dilation angle has a significant growth trend when the bond strength ratio increases. Increasing the bond strength ratio increases the strength of concrete A, thereby changing the failure mode. When the bond strength ratio is relatively small, the asperity can be cut off easily, and the shear dilation phenomenon is not evident. At increased bond strength ratio, the failure near the contact surface is changed from simple shear to wear failure, increasing the peak dilation angle gradually. The fitting relation between peak dilation angle and bond strength ratio is shown as follows:

$$
\psi=a_{1}+b_{1} \sigma_{\mathrm{n}}+b_{2} \sigma_{\mathrm{n}}^{2}
$$

where $a_{1}, b_{1}$, and $b_{2}$ are the fitting coefficients and $\sigma_{\mathrm{n}}$ is the normal stress. The fitting parameters for the peak dilation angle with different bond ratios are shown in Table 3.

3.4. Shear Morphology Damage in Two Medium Materials. The three typical failure modes of the two medium materials under different bond strength and normal stress values are shown in Figure 8. Figure 8(a) shows the distribution of displacement field and microcrack of the sample with a bond strength ratio of 0.25 and normal stress of $1.0 \mathrm{MPa}$. Parts A, $\mathrm{B}$, and $\mathrm{C}$ are selected for feature analysis, and their displacement fields are shown in the right part of Figure 8(a). The figure shows that the failure is mainly concentrated in 

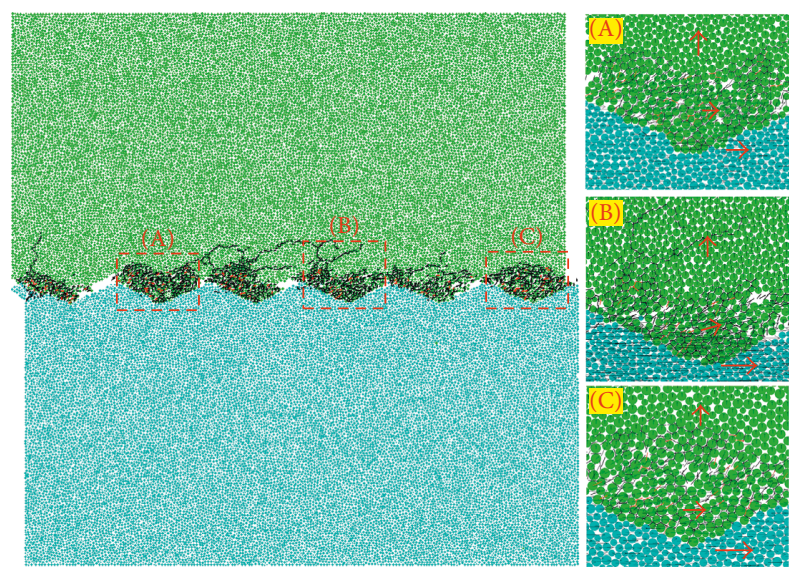

(a)
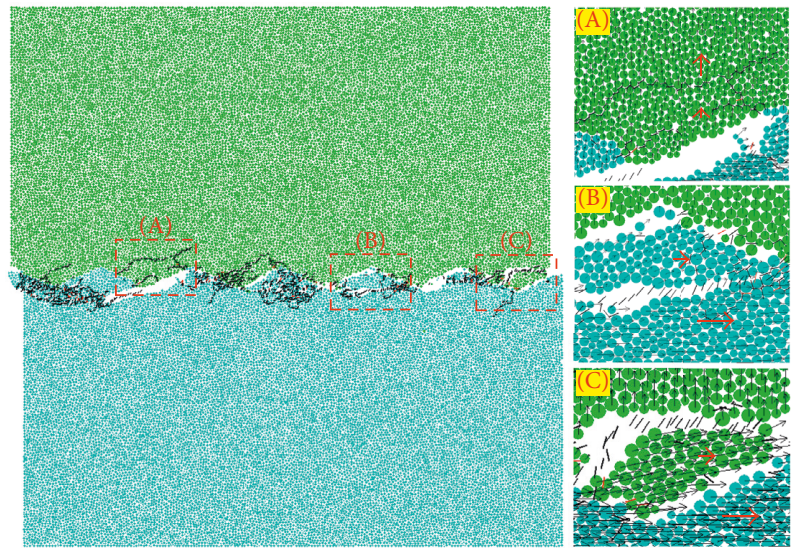

(b)
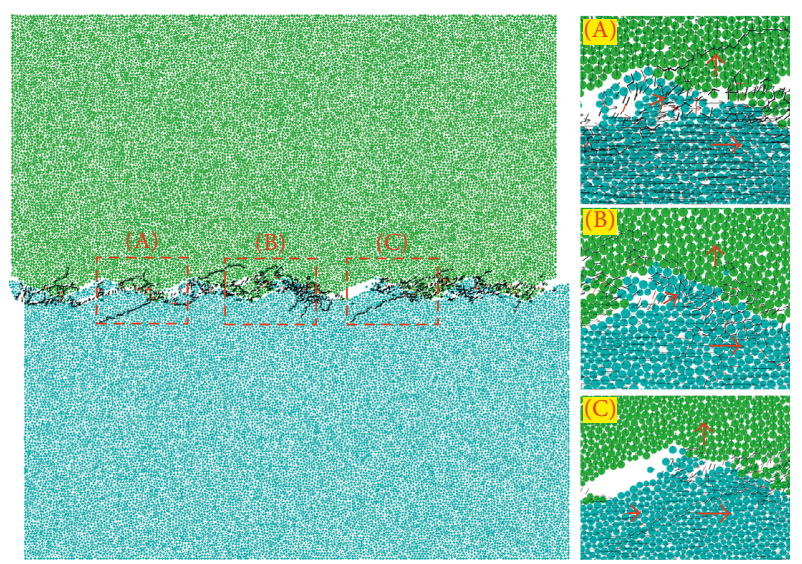

(c)

FIGURE 8: Different kinds of failure modes and distribution of displacement field: (a) bond strength ratio of 0.25 and normal stress of 1.0 MPa, (b) bond strength ratio of 0.75 and normal stress of $1.0 \mathrm{MPa}$, and (c) bond strength ratio of 1.0 and normal stress of 2.0 MPa.

the upper part of the sample. The displacement field of the sheared asperities is observed toward the right direction, while that of other part of the sample is upward through the displacement field shown in the right part of Figure 8(a). The distribution of the displacement vectors with upward
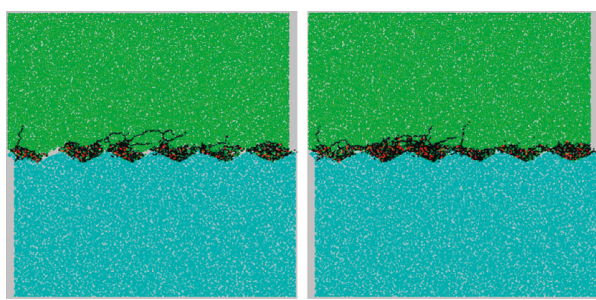

Normal stress of 1.0 MPa Normal stress of 2.0 MPa
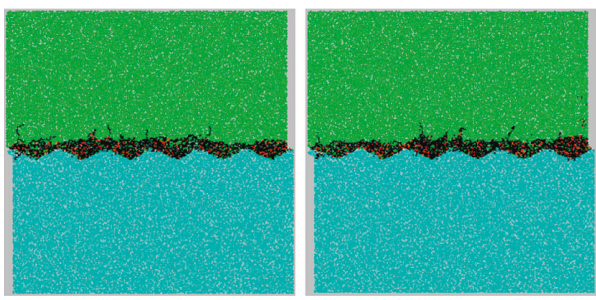

Normal stress of 3.0 MPa Normal stress of 4.0 MPa

(a)
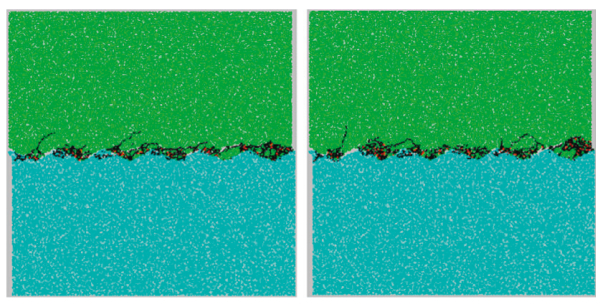

Normal stress of 1.0 MPa Normal stress of 2.0 MPa
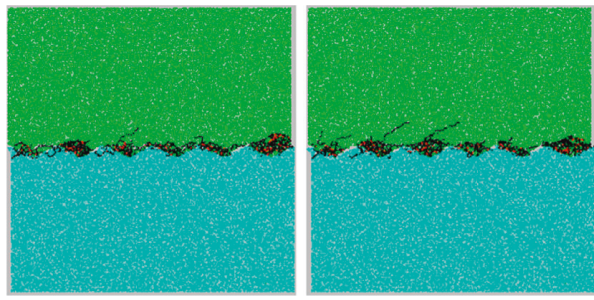

Normal stress of 3.0 MPa Normal stress of 4.0 MPa

(b)

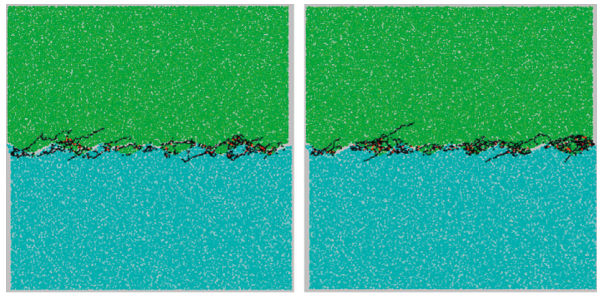

Normal stress of 1.0 MPa Normal stress of 2.0 MPa
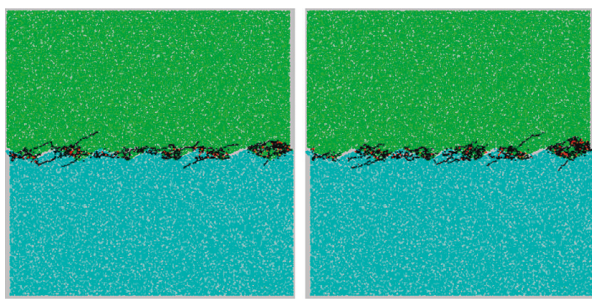

Normal stress of 3.0 MPa Normal stress of 4.0 MPa

(c)

Figure 9: Continued. 


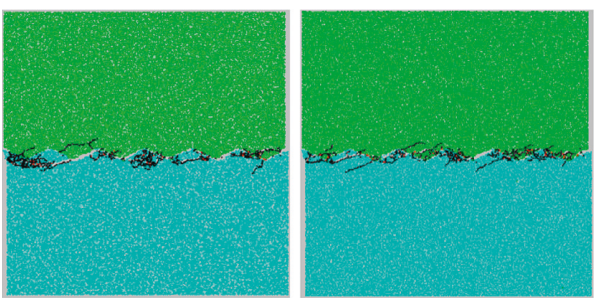

Normal stress of 1.0 MPa Normal stress of $2.0 \mathrm{MPa}$

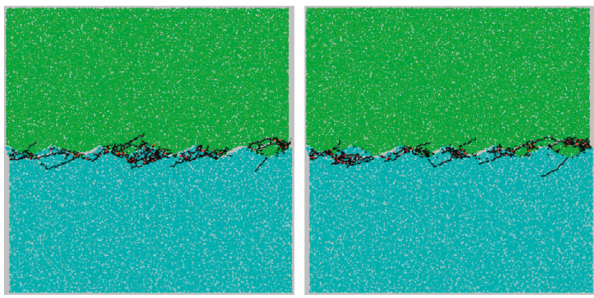

Normal stress of 3.0 MPa Normal stress of 4.0 MPa

(d)

FIGURE 9: Failure modes for specimens with different bond strength ratios under different normal stresses: bond strength ratio of (a) 0.25 , (b) 0.5 , (c) 0.75 , and (d) 1.0 .

movement is uneven, and thus the tension failure is still observed within the model, like in part B. Sperity degradation is present in the upper and lower parts of the sample in the second failure mode (as shown in Figure 8(b)), and the figure illustrated that the upper and lower parts of the model are partially serrated. The green part of the damage is caused by the tensile crack, and thus the sawtooth cut is not generally shear failure and is also a tensile failure. The contact between the upper and lower parts of the model induces the tensile crack at the place of contact, and the tensile cracks propagate continuously, resulting in the fracture of the sawtooth at increased compression under shear load. This kind of failure mode is present in the model with bond strength ratio between 0.5 and 0.75 . Figure 8 (c) shows that, similar to the second failure mode, the third failure mode exhibits damage at the upper and lower two parts. However, tensile cracks near the contact surface are more evident. Consequently, the sawtooth is cut partially rather than completely damaged, as shown in the first and second failure modes.

As mentioned above, the bond strength ratio and normal stress of the upper concrete influence the failure of the model. Figure 9 shows the failure modes of the sample with different bond strength and normal stress values. As shown in Figure 9(a), the failure of samples shows the first failure mode when the upper bond strength is 0.25 . Regardless of the normal stress, the failure of the model is concentrated in the upper part of the model, but nearly no damage occurs in the lower part of the concrete. The damage near the contact surface becomes increasingly apparent at increased normal stress. The model in Figure 9(c) mainly belongs to the second failure mode, and some damages in the upper and lower parts of the contact surface of the model are observed. In addition, the damage of the upper part is more severe than that of the lower part, and some tension cracks are observed

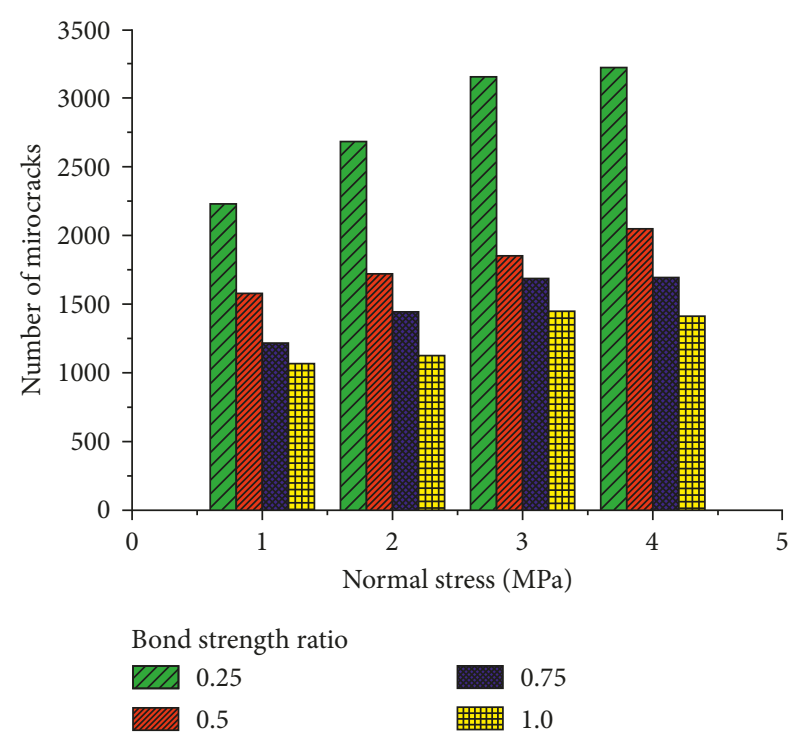

FIGURE 10: Number of microcracks for specimens with different bond strength ratios under different normal stresses.

in the lower part of the model. The failure mode in Figure 9(b) is between the first and second modes. The damages are mainly concentrated in the upper model, and some tensile cracks appear at the upper part of the model when the normal stress increases. However, some wear failures are found in the lower part of the model.

When the bond strength ratio is 1.0, the failure of the model mainly presents a chip-off failure along the interface of the binary-medium model. This failure is the third failure mode, which is a common failure mode of rock joint shear test. In this failure mode, the bond strength of the upper and lower parts of the model and the distribution of natural microcracks are consistent, and the tensile microcracks near the contact surface are also common. Figure 10 shows the distribution and quantity of microcracks in each model with different bond strength ratio and normal stress values. The number of microcracks is the quantitative analysis parameter of the wear and failure of the interface and can represent the size and extent of the damage area in the model after shearing. Figure 10 shows that the number of microcracks shows a significant decreasing trend when the bond strength ratio of the model increases. The decreasing trend indicates that the damage area near the contact surface decreases gradually at increased bond strength ratio, as shown in the failure mode in Figure 9. Meanwhile, owing to the increased normal stress limits, the shear dilation of the model and internal microcrack specimens have obvious growth changes at the same bond strength ratio and increased normal stress. In addition, the damage near the binary-medium interface is more evident. This feature is similar to the failure characteristics of the rock shear experiment.

\section{Conclusions}

The purpose of this paper is to study the shear strength and failure characteristics of a binary-medium interface through the simulation of direct shear. Basing on the discrete element 
numerical analysis method, we establish four coupling models of binary-medium materials with four different bond strength ratios and four different normal stresses. Moreover, the mechanical properties and failure modes are analyzed from the microscopic perspective and on the basis of the results of numerical simulation:

(1) The bond strength of the upper part of the model considerably influences the shear strength parameters of the binary-medium interface. The shear strength parameters apparently increase at increased bond strength ratio. Furthermore, the peak dilation angle shows a decreasing trend at increased normal stress. Conversely, the peak dilation angle shows an increasing trend at increased bond strength.

(2) Three kinds of failure modes are found for the binary-medium model during the direct shear test. The first type of failure mode is shear failure, in which the asperity of the upper concrete has a shearing-off failure. In mode II, some sawtooth experiences shear failure, but some tensile cracks appear on the interface of the binary-medium materials. In mode III, no sawtooth was cut off, mainly exhibiting tensile failure on the interface. When the bond strength ratio is low, the damage or failure is more severe and mainly appears as shear failure. Furthermore, at increased bond strength ratio, the degree of wear and failure near the contact surface is gradually reduced, and the failure mode is mainly manifested as chip-off and tension failure.

\section{Conflicts of Interest}

The authors declare that there are no conflicts of interest regarding the publication of this paper.

\section{Acknowledgments}

This study was funded by the project (51774322 and 51474249) supported by National Natural Science Foundation of China and project (2016CX019) supported by Innovation-Driven Plan in Central South University.

\section{References}

[1] P. Asadollahi and F. Tonon, "Constitutive model for rock fractures: revisiting Barton's empirical model," Engineering Geology, vol. 113, no. 1-4, pp. 11-32, 2010.

[2] M. Bahaaddini, G. Sharrock, and B. K. Hebblewhite, "Numerical investigation of the effect of joint geometrical parameters on the mechanical properties of a non-persistent jointed rock mass under uniaxial compression," Computers and Geotechnics, vol. 49, pp. 206-225, 2013.

[3] Y. Chen, P. Cao, D. Mao, C. Pu, and X. Fan, "Morphological analysis of sheared rock with water-rock interaction effect," International Journal of Rock Mechanics and Mining Sciences, vol. 70, pp. 264-272, 2014.

[4] G. Grasselli, "3D behaviour of bolted rock joints: experimental and numerical study," International Journal of Rock Mechanics and Mining Sciences, vol. 42, no. 1, pp. 13-24, 2005.
[5] M. Boulon, C. Plytas, and P. Foray, "Interface behaviour and prediction of the lateral shaft friction along piles and anchors," Computers and Geotechnics, vol. 5, no. 1, p. 68, 1988.

[6] S. Maghous, D. Bernaud, and E. Couto, "Three-dimensional numerical simulation of rock deformation in bolt-supported tunnels: a homogenization approach," Tunnelling and Underground Space Technology, vol. 31, pp. 68-79, 2012.

[7] L. J. Su, J. H. Yin, and W. H. Zhou, "Influences of overburden pressure and soil dilation on soil nail pull-out resistance," Computers and Geotechnics, vol. 37, no. 4, pp. 555-564, 2010.

[8] A. Haque and J. Kodikara, "A simplified analytical model for predicting the shear behaviour of regular triangular rock/concrete joints under constant normal stiffness," Geotechnique, vol. 62, no. 2, pp. 171-176, 2012.

[9] J. K. Kodikara and I. W. Johnston, "Shear behaviour of irregular triangular rock-concrete joints," International Journal of Rock Mechanics and Mining Science \& Geomechanics Abstracts, vol. 31, no. 4, pp. 313-322, 1994.

[10] H. M. Tian, W. Z. Chen, D. S. Yang, and J. P. Yang, "Experimental and numerical analysis of the shear behaviour of cemented concrete-rock joints," Rock Mechanics and Rock Engineering, vol. 48, no. 1, pp. 213-222, 2015.

[11] M. Bahaaddini, G. Sharrock, and B. K. Hebblewhite, "Numerical direct shear tests to model the shear behaviour of rock joints," Computers and Geotechnics, vol. 51, pp. 101-115, 2013.

[12] N. Barton, "Shear strength criteria for rock, rock joints, rockfill and rock masses: problems and some solutions," Journal of Rock Mechanics and Geotechnical Engineering, vol. 5, no. 4, pp. 249-261, 2013.

[13] J. C. Jaeger, "Shear failure of anisotropic rocks," Geological Magazine, vol. 97, no. 1, pp. 65-72, 1960.

[14] F. D. Patton, "Multiple modes of shear failure in rock," in Proceedings of the 1st International Congress on Rock Mechanics, pp. 509-513, Lisbon, Portugal, September-October 1966.

[15] Y. Li, J. Oh, R. Mitra, and B. Hebblewhite, "A constitutive model for a laboratory rock joint with multi-scale asperity degradation," Computers and Geotechnics, vol. 72, pp. 143151, 2016.

[16] H. Lin, Z. Xiong, T. Liu, R. Cao, and P. Cao, "Numerical simulations of the effect of bolt inclination on the shear strength of rock joints," International Journal of Rock Mechanics and Mining Sciences, vol. 66, pp. 49-56, 2014.

[17] G. Wang, X. Zhang, Y. Jiang, X. Wu, and S. Wang, "Ratedependent mechanical behavior of rough rock joints," International Journal of Rock Mechanics and Mining Sciences, vol. 83, pp. 231-240, 2016.

[18] C. C. Xia, Z. C. Tang, W. M. Xiao, and Y. L. Song, "New peak shear strength criterion of rock joints based on quantified surface description," Rock Mechanics and Rock Engineering, vol. 47, no. 2, pp. 387-400, 2014.

[19] X. Zhang, Q. Jiang, N. Chen, W. Wei, and X. Feng, "Laboratory investigation on shear behavior of rock joints and a new peak shear strength criterion," Rock Mechanics and Rock Engineering, vol. 49, no. 9, pp. 3495-3512, 2016.

[20] R. H. Cao, P. Cao, and H. Lin, "Mechanical behavior of brittle rock-like specimens with pre-existing fissures under uniaxial loading: experimental studies and particle mechanics approach," Rock Mechanics and Rock Engineering, vol. 49, no. 3, pp. 763-783, 2015.

[21] H. Lin, W. Xiong, and Q. Yan, "Three-dimensional effect of tensile strength in the standard Brazilian test considering contact length," Geotechnical Testing Journal, vol. 39, no. 1, pp. 137-143, 2016. 
[22] J. W. Park and J. J. Song, "Numerical simulation of a direct shear test on a rock joint using a bonded-particle model," International Journal of Rock Mechanics and Mining Sciences, vol. 46, no. 8, pp. 1315-1328, 2009.

[23] V. Sarfarazi, A. Ghazvinian, and W. Schubert, "Numerical simulation of the process of fracture of echelon rock joints," Rock Mechanics and Rock Engineering, vol. 47, no. 4, pp. 1355-1371, 2014. 


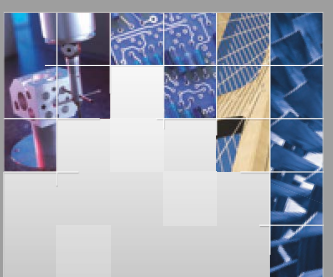

\section{Enfincering}
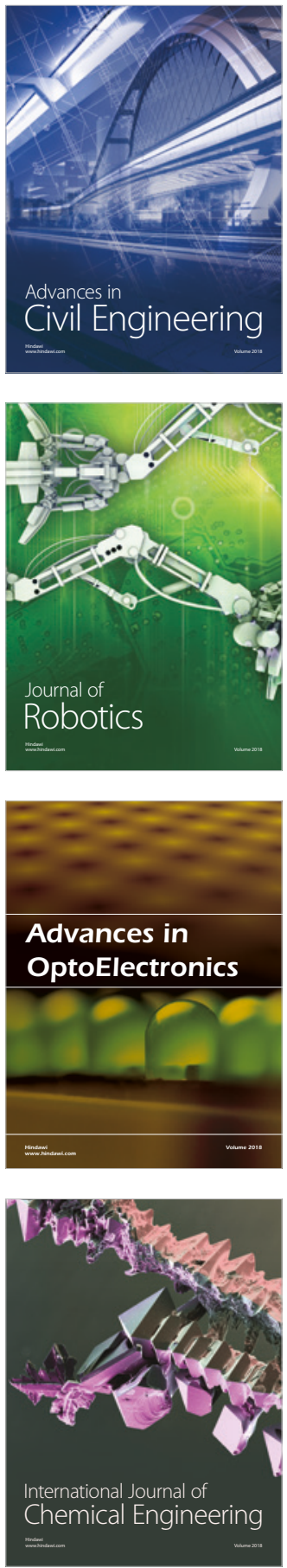

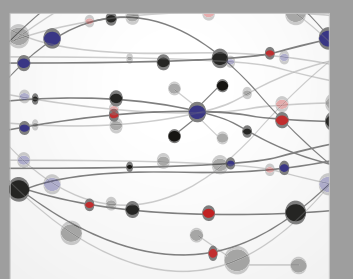

\section{Rotating \\ Machinery}

The Scientific World Journal

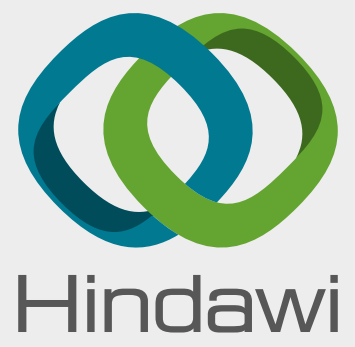

Submit your manuscripts at

www.hindawi.com
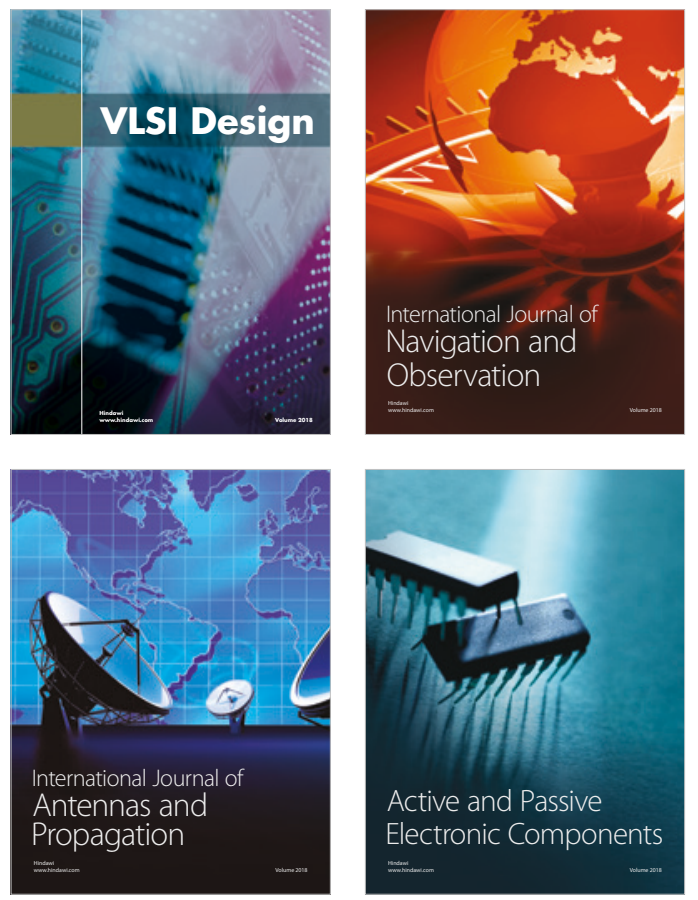
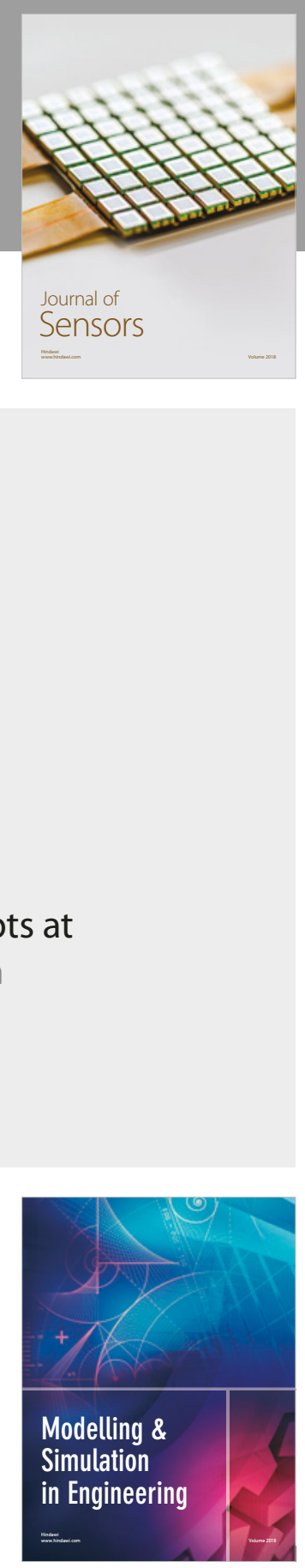

\section{Advances \\ Multimedia}
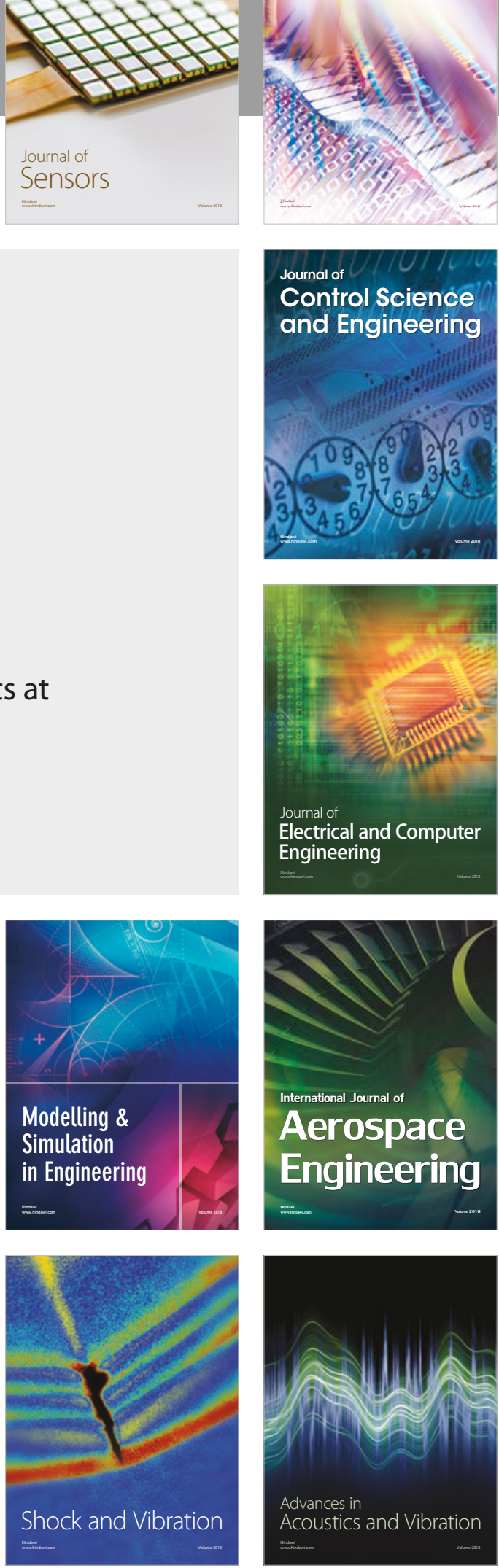